\title{
UV Sensor Based on Polyurethane Foam
}

María Fernanda Stragliotto ${ }^{1,2}$, Giuliana Mosconi ${ }^{1,2}$, Miriam C. Strumia ${ }^{1,2}$, Marcelo R. Romero ${ }^{1,2 *}$, Cesar G. Gomez ${ }^{1,2}$

${ }^{1}$ Universidad Nacional de Córdoba, Facultad de Ciencias Químicas, Departamento de Química Orgánica, Córdoba, Argentina.

${ }^{2}$ Consejo Nacional de Investigaciones Científicas y Técnicas (CONICET), Instituto de Investigación y Desarrollo en Ingeniería de Procesos y Química Aplicada (IPQA), Córdoba, Argentina.

*Corresponding author: E-mail: marceloricardoromero@gmail.com; Phone: +54-03515353869 annex 53343. Edificio de Ciencias II. Haya de la Torre y Medina Allende. (5000) Córdoba. Argentina. 


\begin{abstract}
Ultraviolet light (UV) present in solar radiation has an important effect on the health of human skin. For that reason, we developed a disposable and easy-to-manufacture UV light sensor based on surface color change of a polyurethane (PU) film and examined its performance against radiation received from a lamp or from the sun. The chemical modification after UV exposition and the consequent color variation from light yellow to brown in PU sensor were studied by using RGB image analysis and a reflectance prototype containing an Arduino board. It was found that the photochemical modification of PU films increases absorption of visible radiation in the blue spectrum range. Furthermore, PU surface was more sensitive to UV $(<390)$ than that found in visible light when exposed to sunlight, which was associated with the presence of a chromophore in the material modified. In addition, it was found that the extract obtained from irradiated PU showed its maximum absorption at $309 \mathrm{~nm}$. Hence, the use of this economical light radiation sensor seems to be a very promising tool to determine exposure to UV light from the solar spectrum.
\end{abstract}

Keywords: UV, SOLAR RADIATION, POLYURETHANE, SENSOR, ARDUINO 


\section{Introduction}

Recent studies have found that about $10 \%$ of the US population is intentionally exposed to sunlight at work or during holidays [1]. Although sometimes skin exposure to sunlight has positive effects $[2,3]$, it can be subject to damage produced by UV light from the solar spectrum, which consequently increases the risk of skin cancer [4]. UVA and UVB radiation are the main responsible for biological response, leading, in a few minutes, from sensitization of the skin to erythema, which produces important biological and chemical reactions [3,5-7]. Therefore, an optimal dose of sunlight can be controlled using a simple analytical method to determine the radiation received by skin surface, particularly relevant in medical science and human health. In this sense, numerous dosimeters have been developed for the determination of received UV dose [8-13]. Digital colorimetric analysis is the method most widely used to quantify the color information of a sample image whose data are extracted from red, green and blue (RGB) channels [14-18]. In addition, RGB analysis was used for the identification and quantification of different analytes in food [19]. Solar radiation was determined by using ortho-nitrobenzyl (ONB) modified photocleavable polymer films [20] or composite material based on $\mathrm{TiO}_{2}$ ink and polyvinyl pyrrolidone [21]. Although they have good sensitivity, the synthesis of ONB film is not easy and these materials are generally expensive. By contrast, polyurethane (PU) is a commercial polymer with good mechanical properties and may be easily prepared at low cost and usually used as a thermal insulator. PU also exhibits chemical and morphological changes after exposure to environmental factors such as sunlight, turning this polymer into an ideal material to build a dosimeter. This degradation process has already been observed by Rosu et al., which undertook studies to determine the nature of the chemical modification of the material after photochemical exposure [22]. It was found that absorption of visible light from PU surface changed after oxidation of central methylene group of diphenylmethane diisocyanate (chromophore). In addition, this modification was associated with loss of aromatic rings by reacting with light and oxygen. It was also found that sunlight was responsible for degradation observed in PU surface, mainly involving 
the highest energy radiation. Taking advantage of this phenomenon, in this work, a low-cost and disposable UV sensor based on surface color change of a PU film was built to determine UV radiation. Chemical modification produced onto surface of PU films was studied by using RGB image analysis and a reflectance prototype containing an Arduino board. Moreover, the sensitivity of PU films to solar radiation was studied by reflectance measurement and the surface modification of the PU particles was analyzed by using scanning electron microscopy (SEM).

\section{Experimental}

\subsection{Preparation of PU films}

PU film was produced from a commercial reaction mixture containing 4,4'-diphenylmethane diisocyanate (MDI) (espumafix PU Multi - Afix, China). It was left for polymerization into a silicone container under protection from light exposure for $24 \mathrm{~h}$. Then, PU foam was cut into small fragments with a blade, cooled with liquid air to obtain a brittle material; immediately after, it was pulverized with a mortar. The powder was dried to constant mass in an oven at $40{ }^{\circ} \mathrm{C}$ for $48 \mathrm{~h}$. Subsequently, the samples were sieved between screens of 40 and 20 mesh $(0.42-0.84 \mathrm{~mm})$ [23]. Afterwards, the sample was deposited on the surface of an adhesive paper (STIKO, Argentina) until being completely covered and then cut in a square of $1 \mathrm{~cm}^{2}$. The non-retained material was removed under a current of air. The sample was protected from light and moisture with an aluminum foil and stored until use.

\subsection{Exposure of PU sensor to UV lamp light}

Square PU films were exposed to UV radiation at $254 \mathrm{~nm}$ generated from a lamp (NNI 40/20 35W, UV-Consulting Peshl®, Spain). Lamp was lighted for $10 \mathrm{~min}$ before being used to achieve emission stabilization (Figure S.1). Samples were left $5 \mathrm{~cm}$ beneath the lamp for $0,2,4,7,10$, 15, 20, 25 and $30 \mathrm{~min}$. In other cases, samples were exposed for $20 \mathrm{~min}$ but at different distances from the UV-light source $(5,10,15,20$ and $25 \mathrm{~cm})$. To determine the irradiance and dose received 
by each PU film, a UV-B detector was used. The equipment used was a UV sensor probe based on Silicon Carbide ( $\mathrm{SiC}$ ) detector chip, model "UV Surface" joined to an interface CAN to USB converter model "Digibox" (SgLUX-Germany). All results obtained with this device were registered as irradiance $\left(\mathrm{W} / \mathrm{m}^{2}\right)$. When the samples were exposed to UV of $254 \mathrm{~nm}$ at different distances, the radiant flux that received each surface was calculated from the irradiance measured and Inverse Square Law. All experiments were performed four times for each exposition time or distance, respectively.

\subsection{Exposure of PU sensor to sunlight}

PU films were exposed to solar radiation for 5 days. Each day from $1 \mathrm{pm}$ until $2 \mathrm{pm}$, the polyurethane films were exposed for $60 \mathrm{~min}$ to sunlight directly or through a UV filter (EXUS UVcut L390, Marumi Optical Co. Ltd.). The presence of the filter ensures that the films exposed receive only the visible spectrum from the sun. Thus, wavelengths below $390 \mathrm{~nm}$ were eliminated, while those films directly exposed received total solar radiation. The exposure period was chosen based on the solar zenith. In addition, clear sky conditions were required in each exposure. After receiving sunlight, the reflectance of each sample was measured at least four times with the prototype designed for that purpose. Simultaneously, an illuminance value of a 110-130 klx range was determined with a luxometer (UNI-T model UT383, China), while the UV index was informed by the nearest meteorological center (Ambrosio Taravella International Airport on a weather meteorological service webpage) [24]. All tests were performed at least four times.

\subsection{Image analysis of modified surface of PU films}

Pictures of PU films exposed to UV radiation from the lamp were analyzed using a processing software such as IMAGEJ 1.4g (Wayne Rasband, National Institutes of Health, USA). Firstly, the exposed samples were captured through a digital image taken with a camera of 12 megapixels (Canon, PowerShot SX220 HS, Japan). Later, the image obtained was decomposed into RGB 
components. Then, squares of 9500 pixels containing the image of each exposed film were selected. Average pixel value was calculated between 0 and 255 . This average value was assigned as a signal corresponding to the exposed surface of the film. Samples not exposed to light were used as control and measured as the other films. Samples corresponding to a given exposure time were analyzed four times. Furthermore, pixel blue was named as pxB and all data from this component were normalized $\left(\mathrm{pxB}_{\text {norm }}\right)$ from a relation between an $i$ value $\left(p \times B_{i}\right)$ and the highest value measured $\left(p x B_{\max }\right)$.

\subsection{A prototype for reflectance measurements}

Surface modification of PU films was measured with a prototype where reflectance data of samples before and after UV irradiation were gathered. Figure $1 \mathrm{~A}$ shows a schematic diagram of the prototype built. It has a light source of $3 \mathrm{~mm}$ diameter based on blue light-emitting diode (LED) composed of InGaN with a maximum emission of $470 \mathrm{~nm}$ (A-Bright, China). The detector consists of a photodiode (BPV10, Vishay), where the signal was amplified by the dual operational amplifier TLO82 (STmicroelectronics). The signal from the operational circuit was digitized by an analogue digital converter (AD) from an Atmega328 microcontroller contained in an Arduino UNO board (Italy) and the digitized signal was shown using a 2-line x 16-character LCD display. A free software Arduino 1.8.3 was used to program the microprocessor. Figure 1B shows the arrangement of detector photodiode (a) and LED (b) in reflectance cell. Both components were separated by a mirror (c), whose reflective surface was oriented towards LED. From this arrangement, only the light reflected by the sample surface reached the detector. A time near $0.10 \mathrm{~s}$ was required for the device to record the signal. The reflectance measured for this device was determined in volts (V); however, the parameter used was expressed as a reciprocal of former $\left(\mathrm{V}^{-1}\right)$ and named as reflectance signal (rf), to follow the PU modification as rising function. For comparison with RGB analysis, all data were normalized ( $\left.\mathrm{rf}_{\text {norm}}\right)$ from a relation between an $i$ 
value $\left(r f_{i}\right)$ and the highest signal measured $\left(r f_{\max }\right)$. For detailed information on the reflectance prototype, see supplementary data (video S.2).

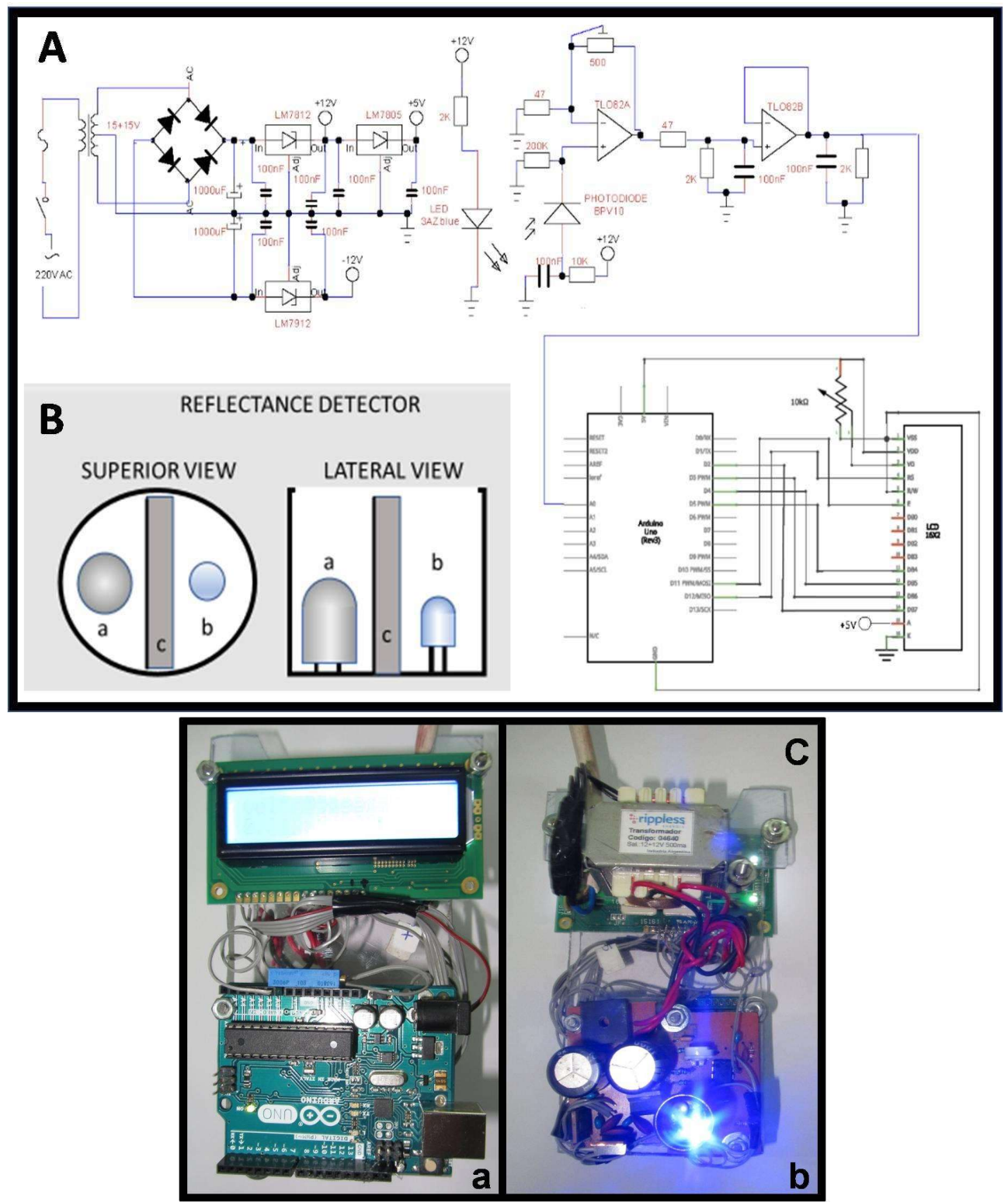

Figure 1. A) Schematic circuit of reflectance detector for PU. B) Spatial location of photodiode (a), led diode (b) and mirror (c) in reflectance detector. C) Photograph of home device for reflectance measurement (a) Microcontroller and display side. b) Detector, amplifier and power source side

\subsection{Stability study of irradiated PU films}


Surface stability of PU sensor was studied to know its ability to maintain the information received from modification produced by radiation. PU film was irradiated following the procedure explained above and its surface was analyzed by the reflectance device. Subsequently, each PU sensor was kept protected from light at $25^{\circ} \mathrm{C}$ under aluminum foil. Surface reflectance of PU sensor was measured again after 15,30 and 60 storage days after irradiation.

\subsection{Film characterization by Infrared spectroscopy}

Surface of films irradiated under UV light and those exposed to sunlight were studied by attenuated total reflectance Fourier Transform Infrared Spectroscopy (FTIR-ATR). Samples were measured with a Thermo Scientific Nicolet IZ10 spectrometer. A zinc selenide ATR crystal with an incident beam at $45^{\circ}$ was used in a wavenumber range of 4000 to $600 \mathrm{~cm}^{-1}$ at $4 \mathrm{~cm}^{-1}$ resolution for 32 scans with Omnic 8 (Nicolet) data processing.

\subsection{Film characterization by scanning electron microscopy}

The surface morphology of PU films before and after exposure to solar radiation was characterized by scanning electron microscopy (SEM). SEM was performed using a Sigma FESEM from the Electron Microscopy Laboratory (LAMARX), FAMAF, Universidad Nacional de Córdoba, Argentina. Each sample was sputter-coated with gold (Au).

\subsection{Irradiated PU characterization by UV-vis spectroscopy}

PU foam samples were prepared with a spherical shape of near $5 \mathrm{~cm}$ of diameter, and then, exposed to sunlight for 1 month. After that, each sample was scraped and $18 \mathrm{mg}$ of PU particles were put in contact with $2 \mathrm{~g}$ of dimethyl sulfoxide (DMSO) for $24 \mathrm{~h}$. Later, a 0.44 wt.-\% of modified $\mathrm{PU}$ extract $\left(\mathrm{PU}_{\mathrm{mod}}\right)$ solution was obtained. Different solutions from dilution of soluble extract in distilled water $(1 / 600,1 / 343,1 / 240,1 / 150$ and 1/120) were prepared. The UV-vis spectra of 
modified PU extract solutions were recorded with a Shimadzu UV-1800 spectrophotometer (Japan) in a wavelength range of $200-1000 \mathrm{~nm}$.

\section{Results and discussion}

\subsection{UV irradiation of samples}

PU films were irradiated with a radiant flux of $188 \mathrm{~W} \cdot \mathrm{m}^{-2}$ from lamp of UV light for different times. All samples received doses of $0.0,2.2,4.5,7.9,11.3,16.9,22.6,28.2$ and $33.8 \mathrm{J.cm}^{-2}$, respectively. Figure 2 shows that the non-irradiated sample had a pale yellowish color. However, as exposure time increases, the sample turns dark where the original color of PU surface shifts toward brown.

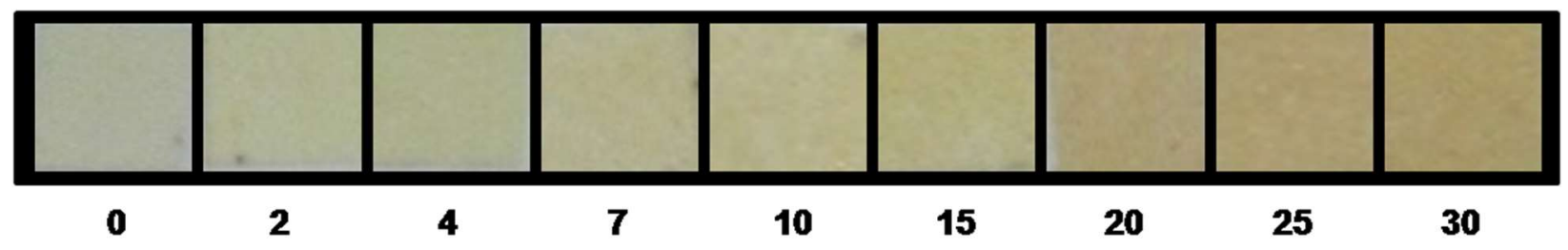

Figure 2. Photograph of PU films after different exposition times (minutes) under UV light.

In addition, chemical modification produced onto surface of PU films after irradiation was measured with FTIR-ATR spectroscopy. A similar study was already performed by Rosu et. al., reporting several characteristic bands corresponding to specific groups formed after photochemical decomposition [22]. Among the most relevant signals are those at 1730 and 1705 $\mathrm{cm}^{-1}$ corresponding to stretching vibration of carbonyl group of ester and urethane group, respectively. Figure 3 shows that the carbonyl signal of ester group increases while the urethane band decreases as a function of light exposition time. A superficial photochemical degradation from amide to ester group (Photo-Fries rearrangement) took place [22]. Other bands corresponding to ring of $\mathrm{C}=\mathrm{C}$ stretching mode and its bending vibration in-plane at 1595 and 1506 
$\mathrm{cm}^{-1}$ were observed, respectively. This phenomenon is attributed to the photo-oxidation of methylene group of $\mathrm{MDI}$, leading to the conversion of aromatic rings to highly conjugated quinones [22]. In addition, the methyl group shows a band at $1370 \mathrm{~cm}^{-1}$ corresponding to the bending vibration, which is increased from decomposition of alkyl ester structures present in commercial PU [22].

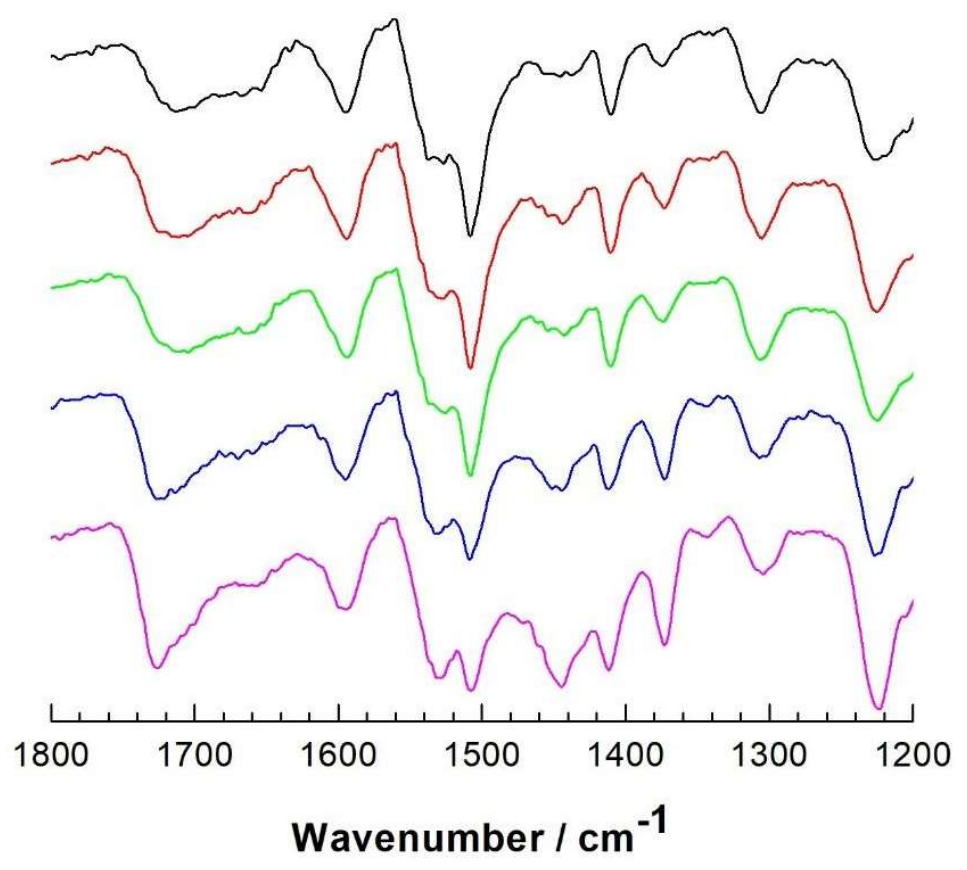

Figure 3. FTIR-ATR spectra of PU samples obtained after UV irradiation time such as 0 (black), 2 (red), 5 (green), 15 (blue) and 20 min (pink).

In addition, the chemical change which occurred onto PU film surface after exposure to light at different times was evaluated from RGB image analysis. Figure 4A shows the original images as a reference scale and their RGB components obtained from digital photography of irradiated films. It is clearly observed that the major change is mainly shown from the blue component of captured images, whose absorption value increases with exposure time. Moreover, Figure 4B shows average pixel value for the red, green and blue components obtained from image of PU film after UV light exposure at different times. Although the signals of the green and red components show no clear dependence, the pixel value of the blue component linearly decreases as exposure time 
increases until $20 \min \left(R^{2}=0.996\right)$. Then, a constant value is reached. This phenomenon was associated with the fact that the photodegradation of PU film surface took place with a constant rate until $20 \mathrm{~min}$, and then total surface conversion was achieved.
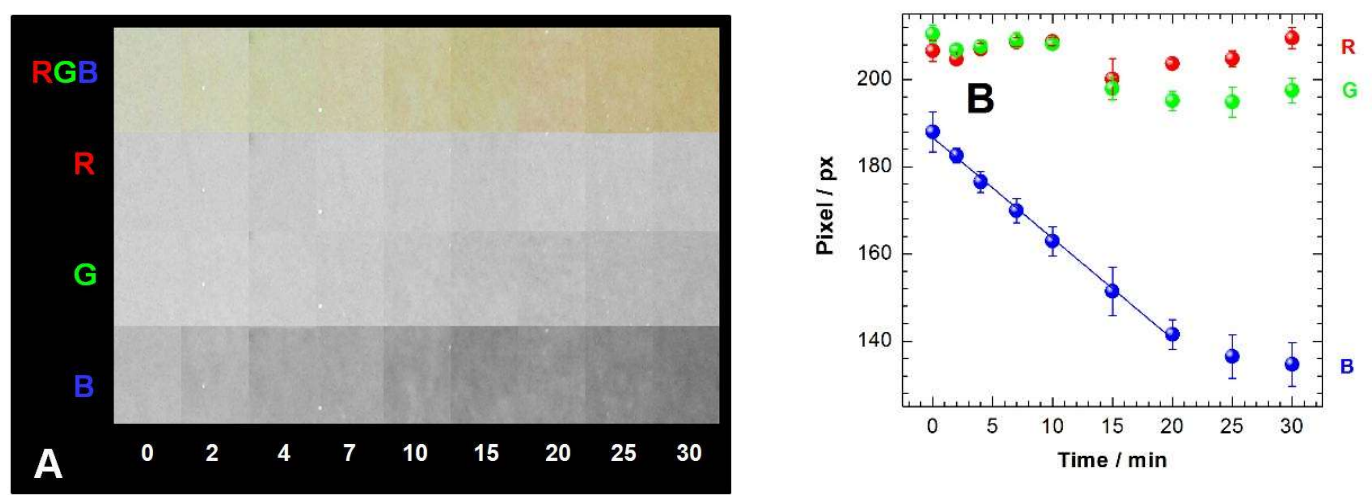

Figure 4. RGB analysis of PU films exposed to UV light. (A: left) Comparison between color image and red (R), green (G) and blue (B) components of PU films at different exposure times. (B: right) Pixel variation of $R, G$ and $B$ components as a function of UV exposure time.

\subsection{Performance of reflectance prototype}

Operation of this device is based on rf measurement when the surface of PU film is stimulated by blue light emitted from a photodiode (Figure 1B). Figure $5 \mathrm{~A}$ shows the reflectance of irradiated PU films against exposure time. It is found that reflectance signal increases until 20 min, reaching a constant value. This result agrees with those data about absorption performance of blue component as described above. Therefore, those data obtained from RGB image processing and determination of their reflectance were compared as a function of photodegradation time. Thus, for comparison, RGB data and reflectance signal were normalized as $p \times B_{n o r m}=\left(p x B_{i} / p x B_{\max }\right)^{-1}$ and $\mathrm{rf}_{\text {norm }}=\left(\mathrm{rf}_{\mathrm{i}} / \mathrm{ff}_{\max }\right)$, respectively. Figure $5 \mathrm{~B}$ shows the profile of $\mathrm{rf}_{\text {norm }} \mathrm{vs}$ pxB $\mathrm{B}_{\text {norm }}$ curve where a good correlation between these parameters is observed, which supports the fact that both signals are sensitive to chemical modification. It is also found that the linear adjustment has a slope of $(1.85 \pm 0.09)$, intercept $(-0.8 \pm 0.1)$ with a determination coefficient of $r^{2}=0.991$, showing that reflectance has a sensitivity higher than that found in RGB analysis. 

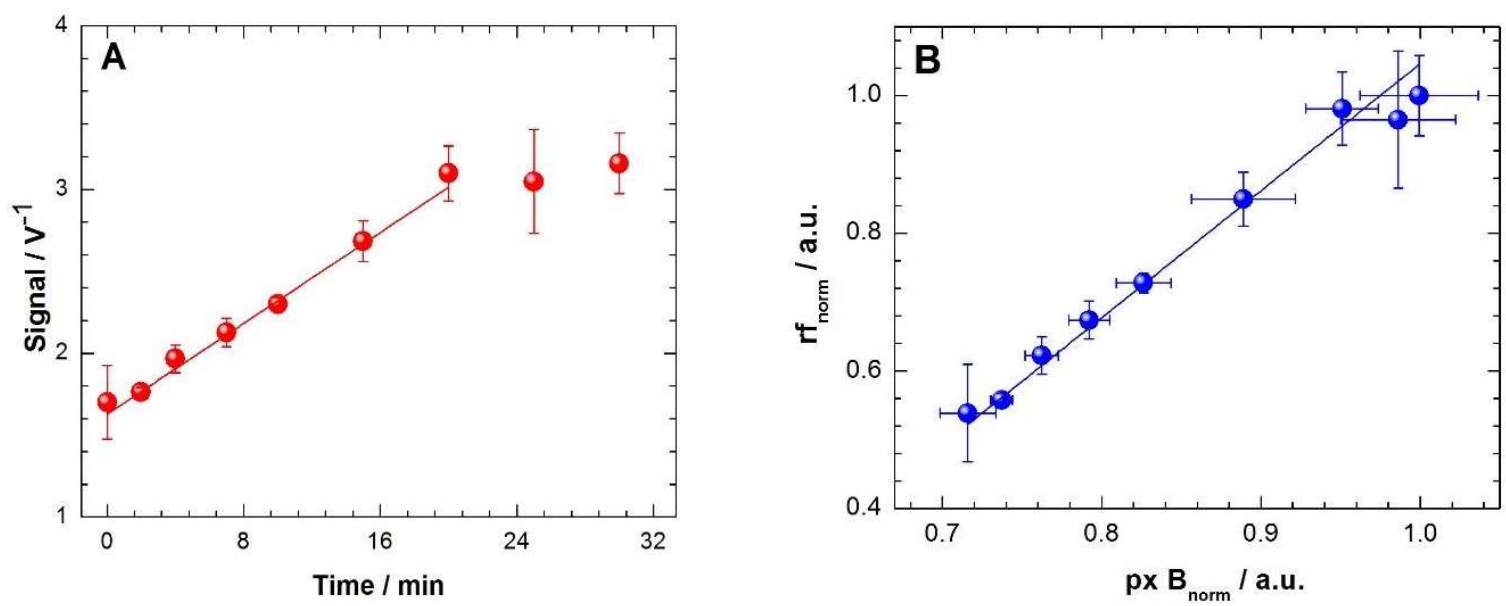

Figure 5. A) Reflectance signal measured as a function of exposure time. B) Correlation between the blue component of RGB analysis and the reflectance measured.

\subsection{Reflectance of irradiated PU films at different UV light intensities}

Sensitivity of PU films exposed to different intensities of UV light at $254 \mathrm{~nm}$ was determined by rf measurement. For this, samples were exposed at different distances from UV lamp for $20 \mathrm{~min}$, where PU surfaces received radiant fluxes about 5 to $200 \mathrm{~W}$. $\mathrm{m}^{-2}$. Figure 6 shows the signal of reflectance obtained at different intensities. A linear relationship was found between both variables with a determination coefficient of 0.96 and a proportionality constant of $6.1 \times 10^{-3}$ $\mathrm{V}^{-1} \mathrm{~W} \cdot \mathrm{m}^{-2}$ 


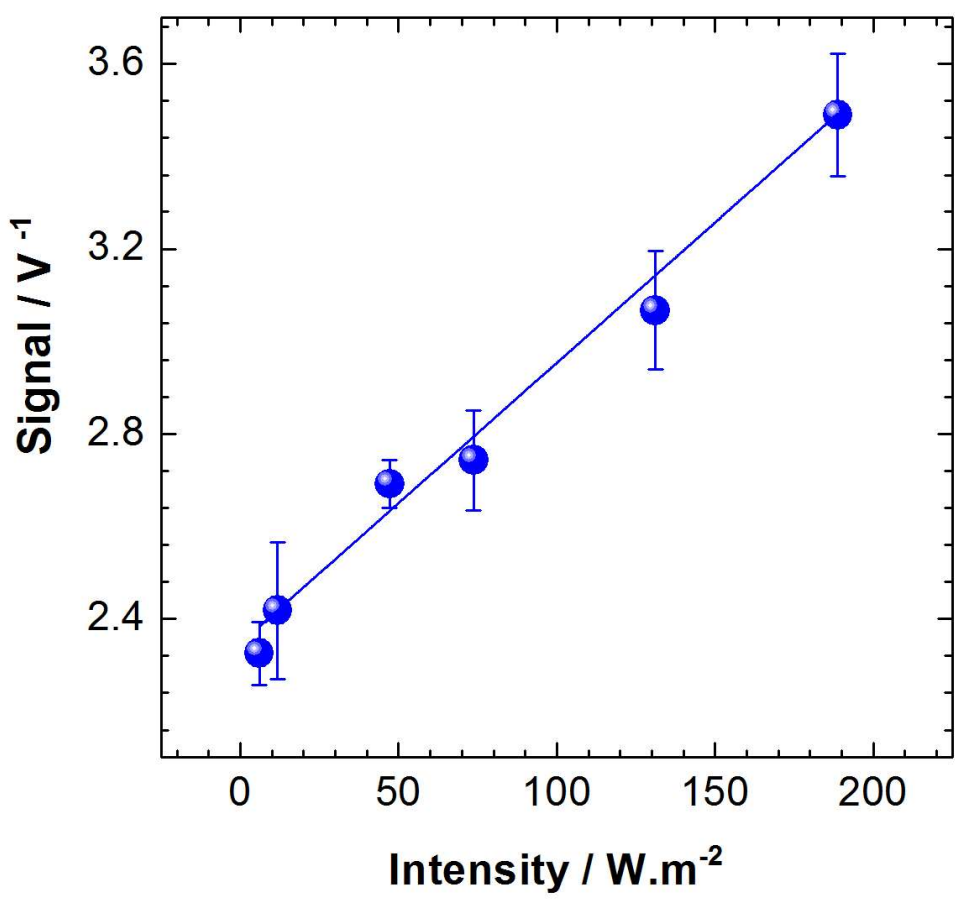

Figure 6. Reflectance signal measured as a UV intensity function for PU films after 20 min of exposure.

\subsection{Stability of PU films exposed to UV light as a function of storage time}

After photochemical modification of PU film took place, chemical stability of surface film was evaluated by reflectance measurements at $0,15,30$ and 60 storage days. Figures $5 \mathrm{~A}$ and 7 show the reflectance response of the films as a function of exposure time. For a storage time of 0 day, the sensitivity found for this sensor was $(1.63 \pm 0.03)$ of $\mathrm{V} \cdot \mathrm{min}^{-1}$, while a detection limit of $1.5 \mathrm{~min}$ of exposure time was determined (Figure 5A). However, 15 days after exposure, the sensitivity of PU films decreased near $25 \%$ in reference to the initial value, while the linear range was maintained (Figure 7A). In addition, the determination coefficient obtained from data showed no change. On the other hand, Figure 7B shows that when reflectance data were recorded after 30 storage days, the slope decreased close to $31 \%$ in relation to its initial value, while the determination coefficient decreased near 0.95 . This effect is more pronounced when the films 
were studied for 60 storage days (Figure $7 C$ ). It was found that sensitivity decreased close to $38 \%$ of its initial value, showing a shorter linear range and its determination coefficient diminished to $r^{2}=0.91$. Although the signal of sample decreased along with a longer storage time after photochemical modification, they maintained a linear correlation at least for 30 storage days.
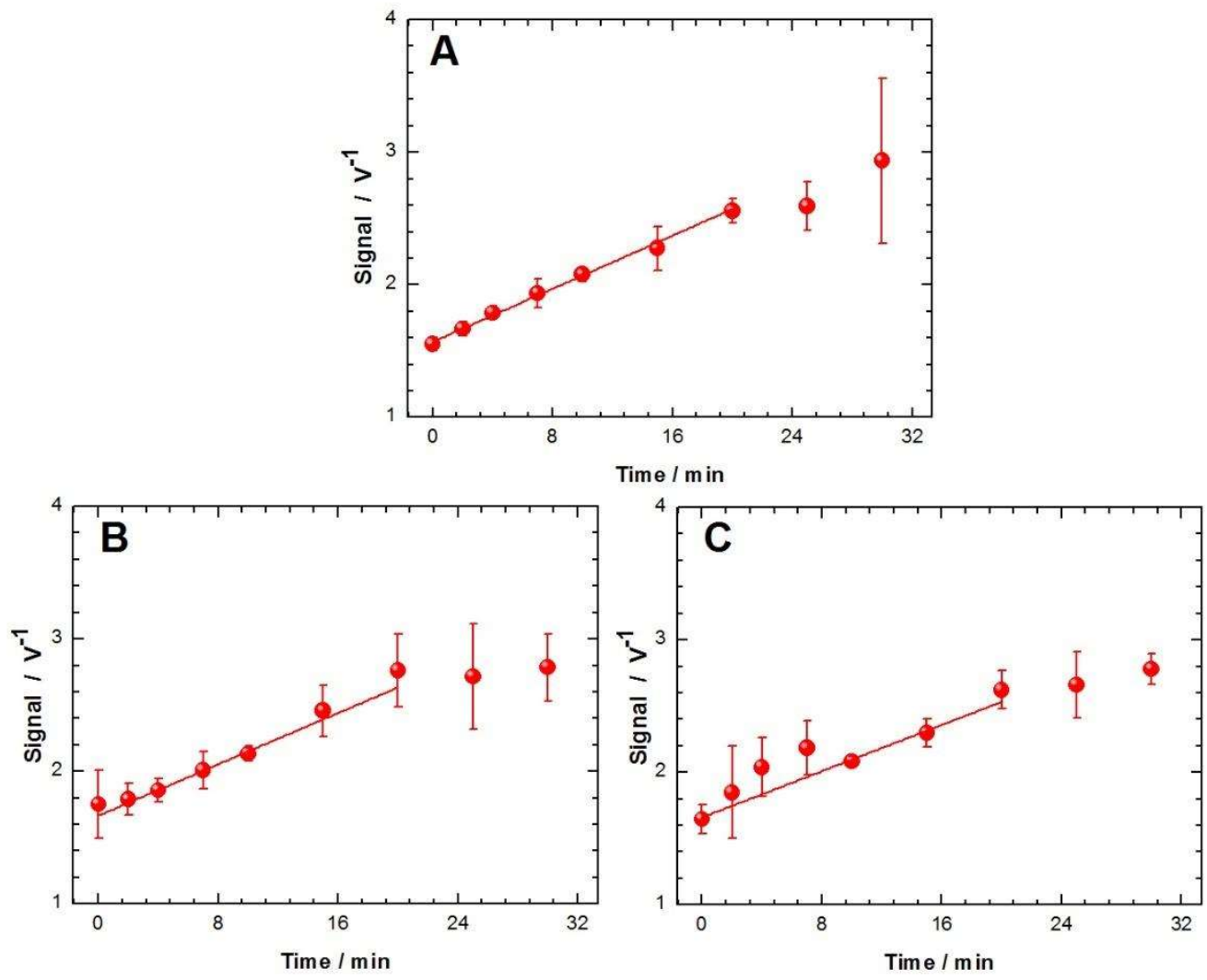

Figure 7. Stability study of PU films exposed to UV light for 15 (A), 30 (B) or 60 (C) storage days.

\subsection{Sunlight influence on photochemical modification of PU sensor}

Figure 8 shows the performance of reflectance as a function of exposure time for samples exposed to solar radiation in the presence or absence of UV filter, which prevents radiation from wavelengths lower than $390 \mathrm{~nm}$. In this measurement, an UV index between 11 and 12 (very high) was reported. In Figure 8 it is also observed that films exposed to solar radiation in the presence of UV filter show no significant change against films directly exposed to sunlight. This 
behavior suggests that films respond to UV light from sunlight with wavelengths below $390 \mathrm{~nm}$. The exposed films show a significant increase of the signal as a time function. The tendency seen in the directly exposed films is attributed to a gradual chemical modification of the sites available onto surface of PU particles.

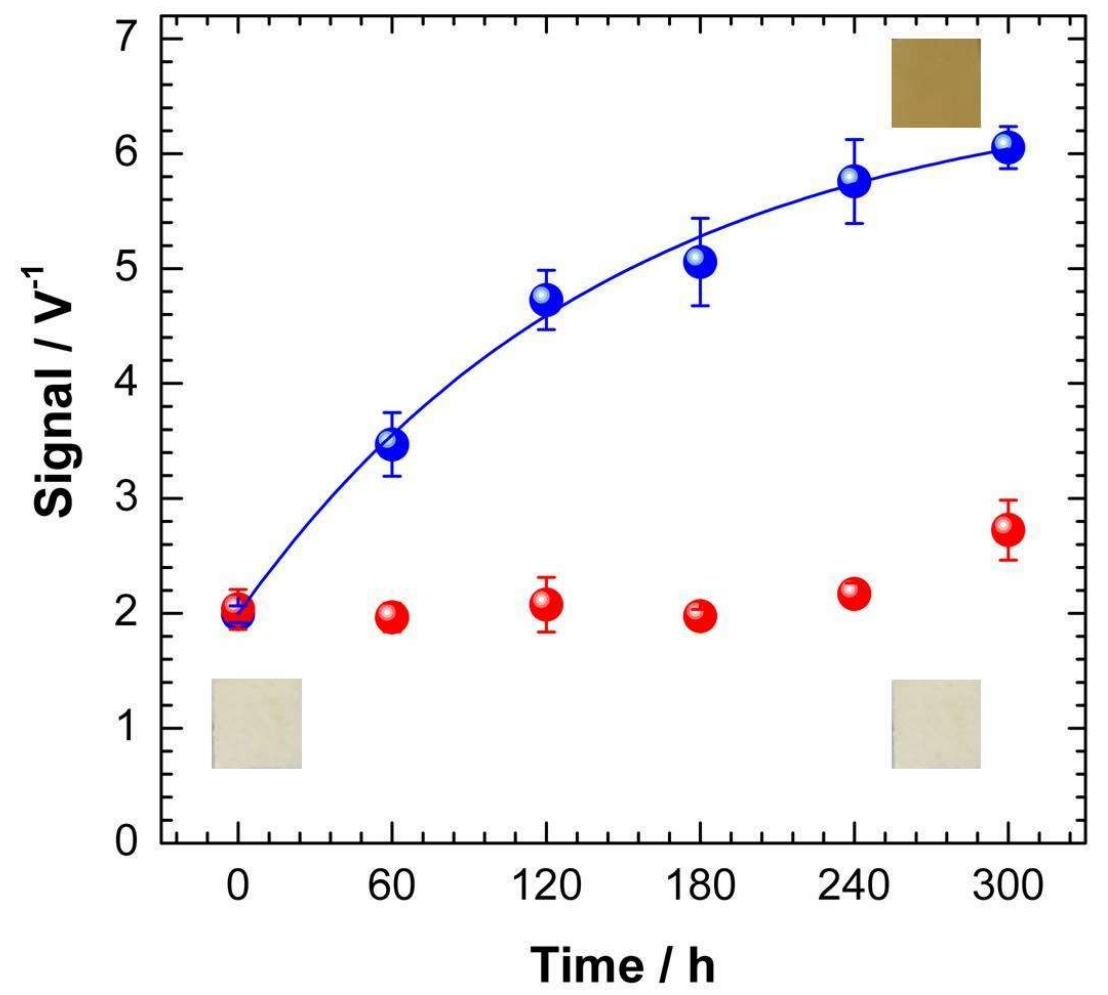

Figure 8. Profile of reflectance signal from surfaces of PU films exposed to sunlight with (red) and without (blue) UV filter as an exposure time.

Equation 1 was used as a mathematical expression to describe the signal variation as a function of exposure time.

$$
\operatorname{Signal}\left(V^{-1}\right)=10 A-7 A^{(x+1)}
$$

where $\mathrm{x}$ was the exposure time, with a parameter $\mathrm{A} / \mathrm{V}^{-1}=0.664 \pm 0.008$ and a determination coefficient $R^{2}=0.99879$ was estimated. Then, PU films before and after exposure to solar radiation were characterized by scanning electron microscopy (SEM). Figure 9 depicts SEM 
micrographs corresponding to the surface of PU films where magnification increases from left to right. On the left, the top layer of the film seems to be completely covered with PU particles, attaining a fairly uniform final surface. On the top micrographs exhibit the surface of PU films exposed to sunlight under UV filter protection; on the bottom PU films are modified by UV sun radiation. Clearly, the micrographs with the highest magnification allow visualizing the incipient chemical modification that takes place onto the surface of PU film without UV filter after sunlight exposure. Small and dark dots are homogeneously distributed onto the surface of modified PU films, where chemical modification takes place. No appreciable loss of material can be found from the superficial morphology, which suggests that sensitivity is probably based on local chemical modification. This behavior had already been corroborated by FT-IR-ATR.

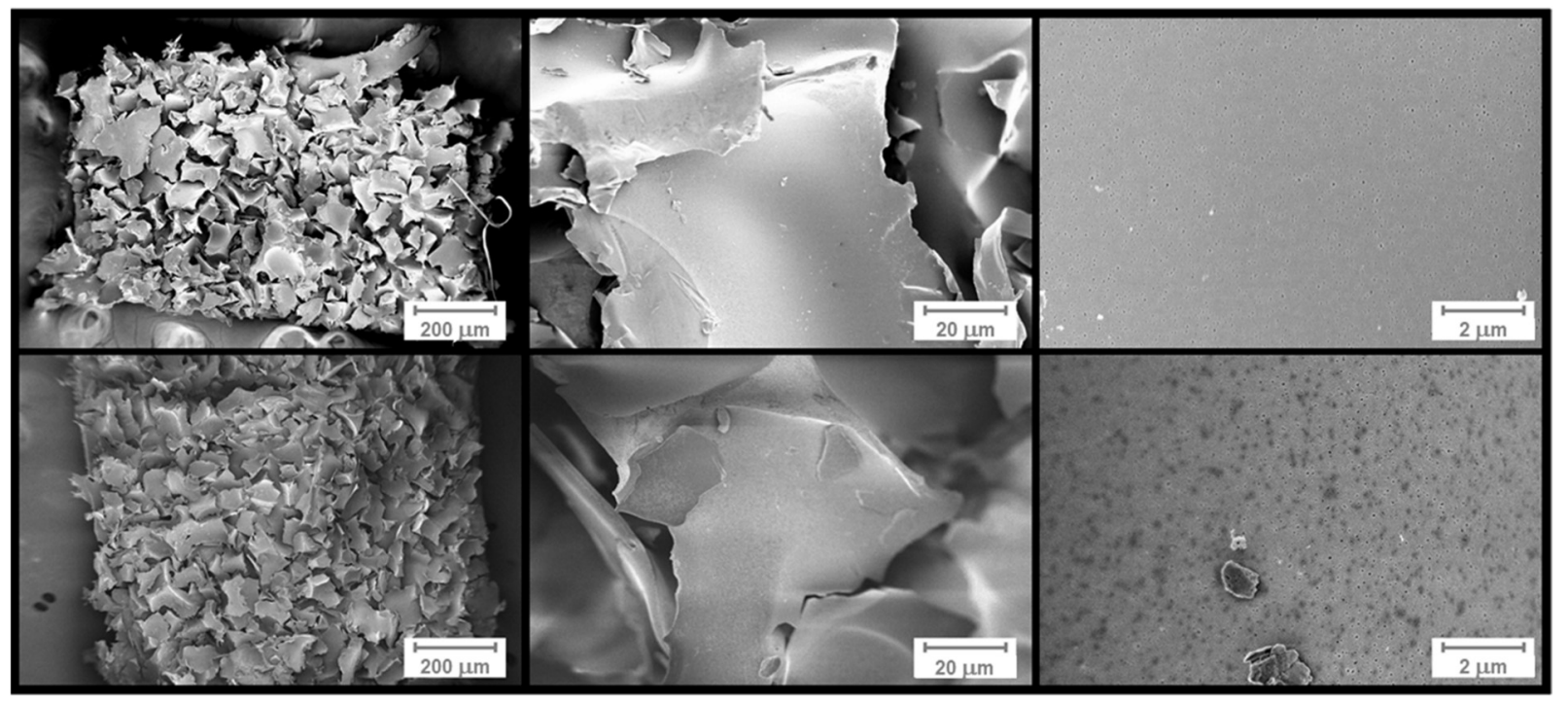

Figure 9. SEM micrographs of the surface of PU films after exposure to sunlight with (bottom) or without (up) UV filter. Magnification of 50,500 or $5 \mathrm{kX}$ is reported inside micrographs.

\subsection{Spectral characterization of irradiated PU}

It is known that the direct and circumsolar irradiance that reaches the surface of the earth has an appreciable intensity higher than $5 \times 10^{-4} \mathrm{~W} \cdot \mathrm{m}^{-2} \mathrm{~nm}^{-1}$ at wavelengths above $300 \mathrm{~nm}$ [25]. In this sense, when PU films were exposed to solar radiation, superficial changes were observed. 
However, when this polymer was exposed to filtered sunlight (without radiation below $390 \mathrm{~nm}$ ), the modification of PU surface did not occur. This behavior suggests that the radiation responsible for the modification of PU surface corresponds to UV light absorbed in the wavelength range of 300-390 $\mathrm{nm}$. Therefore, an active molecule that absorbs UV radiation in this region is expected, related to the degradation product of irradiated PU. In this sense, the soluble extract obtained from irradiated PU (PU mod $)$ by sunlight was characterized by UV-Vis spectroscopy. Figure 10 shows UV-Vis spectral curves of this dilution in the wavelength range of $200-700 \mathrm{~nm}$. The profile of the absorption spectrum of the extract shows a high absorption below $250 \mathrm{~nm}$ and a shoulder at $309 \mathrm{~nm}\left(I_{\max }\right)$, which is suggested as the main absorption band of the solar UV radiation. Beyond this wavelength, the spectrum has an absorption tail extended to visible light range. This phenomenon explains the variation of the blue component (RGB) and reflectance of blue light as PU films are irradiated. In addition, a linear relationship between absorbance and $\mathrm{PU}_{\bmod }$ concentration at $I_{\max }$ was determined (inset of Figure 10). Furthermore, the percentage of absorptivity ( $e_{\text {percent }}$ ) was determined from the slope of the linear adjustment, obtaining a determination coefficient of 0.998 , and $e_{\text {percent }}$ value of $(207 \pm 5) \% \mathrm{wt}^{-1} . \mathrm{cm}^{-1}$. 


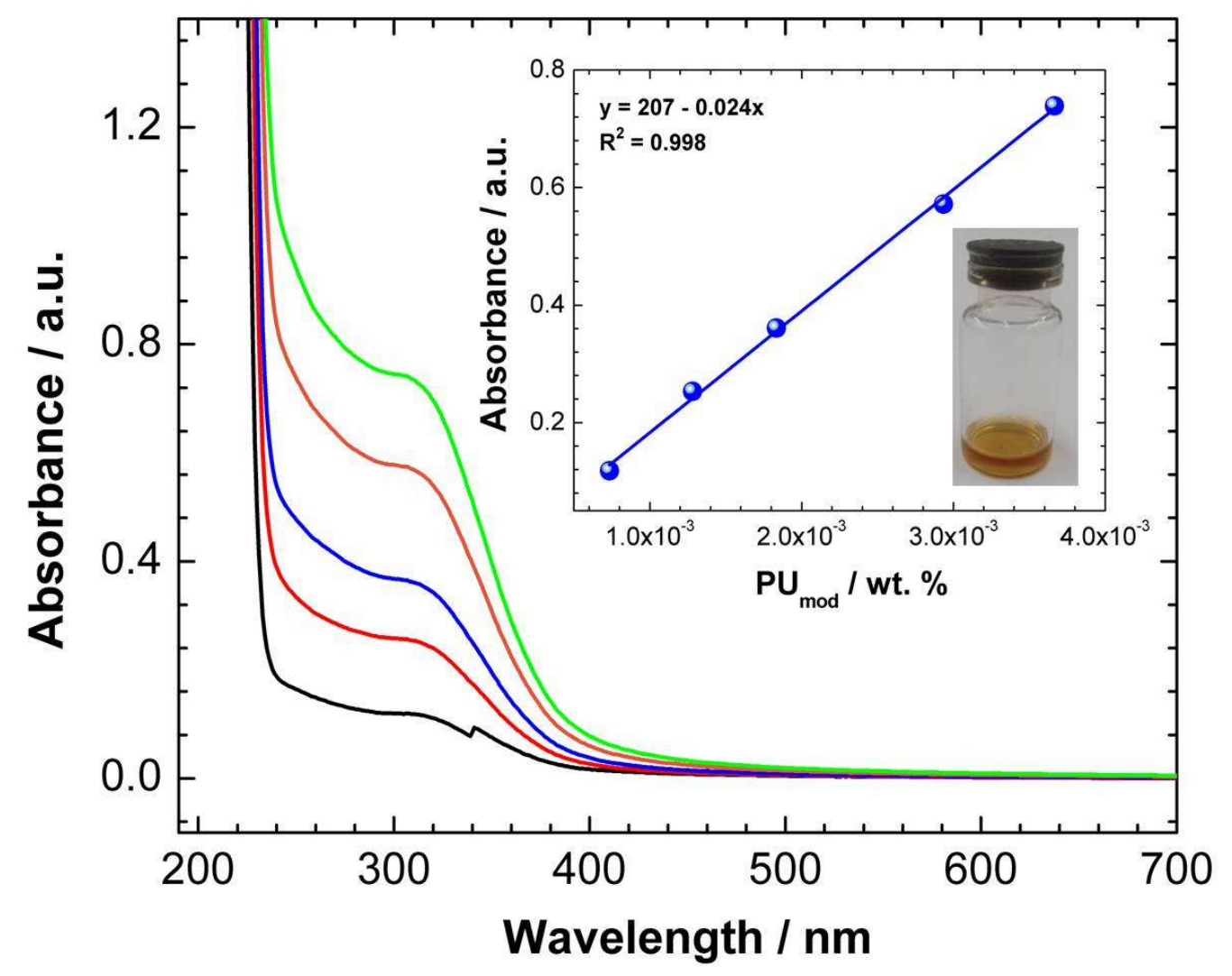

Figure 10: UV-visible spectra corresponding to $\mathrm{PU}_{\bmod }$ dilutions. Inset: Calibration curve determined at $309 \mathrm{~nm}$ for $\mathrm{PU}_{\text {mod. }}$ Photograph of a vial with a $3.7 \times 10^{-3}$ wt.\% of $\mathrm{PU}_{\bmod }$ solution.

\section{Conclusions}

PU films were obtained from a commercial mixture containing MDI. Surface PU films showed color change in the range of yellow as a response to the UV radiation received. In addition, the development of a device based on reflectance value with Arduino technology was proposed as a useful technique. Thus, according to the tests conducted we could confirm that PU films exposed to solar radiation were sensitive only to a wavelength shorter than $390 \mathrm{~nm}$, which belongs to the UV radiation of the solar spectrum. It was also found that UV sensor was very stable and adequately kept the information at least for 30 storage days. The surface of PU film modified by 
irradiation was also characterized by SEM. Therefore, the use of this photosensitive surface as an economical sensor of light radiation seems a promising tool to control the UV radiation received. Moreover, this kind of sensing surface is being evaluated as a future work to build similar sensors from recycled PU.

\section{Acknowledgements}

María Fernanda Stragliotto, PhD., thanks CONICET for her postdoctoral fellowship. In addition, the authors are grateful for the financial support received from CONICET through the projects PIP 11220110100499CO and PIP 11220150100344CO, FONCYT (PICT-2015-2477) and SeCyTUNC (30820150100348CB). The authors also wish to acknowledge language assistance by Prof. Carolina Mosconi and thank Prof. José Alberto Riveros for technical assistance by SEM. Furthermore, the authors also thank Juan Atea from LIADE laboratory for his assistance in irradiance measurements.

\section{References}

[1] M.L. Shoemaker, Z. Berkowitz, M. Watson, Intentional outdoor tanning in the United States: Results from the 2015 Summer ConsumerStyles survey, Prev. Med. (Baltim). 101 (2017) 137-141. doi:10.1016/j.ypmed.2017.06.003.

[2] G.P. Guy, M. Watson, A.B. Seidenberg, A.M. Hartman, D.M. Holman, F. Perna, Trends in indoor tanning and its association with sunburn among US adults, J. Am. Acad. Dermatol. 76 (2017) 1191-1193. doi:10.1016/j.jaad.2017.01.022.

[3] L. Wainwright, A. V. Parisi, N. Downs, Dosimeter based on 8-methoxypsoralen for UVA exposures over extended periods, J. Photochem. Photobiol. B Biol. 148 (2015) 246-251. doi:10.1016/j.jphotobiol.2015.04.012.

[4] B. Petersen, M. Triguero-Mas, B. Maier, E. Thieden, P.A. Philipsen, J. Heydenreich, P. Dadvand, H. Maier, M.M.L. Grage, G.I. Harrison, A.W. Schmalwieser, M.J. 
Nieuwenhuijsen, A.R. Young, H.C. Wulf, Sun behaviour and personal UVR exposure among Europeans on short term holidays, J. Photochem. Photobiol. B Biol. 151 (2015) 264-269. doi:10.1016/j.jphotobiol.2015.08.022.

[5] L. Wainwright, A. V. Parisi, N. Downs, Dual calibrated dosimeter for simultaneous measurements of erythemal and Vitamin D effective solar ultraviolet radiation, J. Photochem. Photobiol. B Biol. 157 (2016) 15-21. doi:10.1016/j.jphotobiol.2016.02.003.

[6] A. Bérces, A. Fekete, S. Gáspár, P. Gróf, P. Rettberg, G. Horneck, G. Rontó, Biological UV dosimeters in the assessment of the biological hazard from environmental radiation, J. Photochem. Photobiol. B Biol. 53 (1999) 36-43. doi:10.1016/S1011-1344(99)00123-2.

[7] P. Rettberg, G. Horneck, Biologically weighted measurement of UV radiation in space and on Earth with the biofilm technique, Adv. Sp. Res. 26 (2000) 2005-2014. doi:10.1016/S0273-1177(00)00175-7.

[8] M. Kozicki, E. Sąsiadek, S. Kadłubowski, M. Dudek, P. Maras, A. Nosal, M. GazickiLipman, Flat foils as UV and ionising radiation dosimeters, J. Photochem. Photobiol. A Chem. 351 (2018) 179-196. doi:10.1016/j.jphotochem.2017.10.028.

[9] M. Kozicki, E. Sassiadek, UV dosimeter based on polyamide woven fabric and nitro blue tetrazolium chloride as an active compound, Radiat. Meas. 46 (2011) 1123-1137. doi:10.1016/j.radmeas.2011.06.060.

[10] M. Kozicki, E. Sąsiadek, I. Karbownik, W. Maniukiewicz, Doped polyacrylonitrile fibres as UV radiation sensors, Sensors Actuators, B Chem. 213 (2015) 234-243. doi:10.1016/j.snb.2015.02.087.

[11] A. Amar, A. V. Parisi, Optical properties of a long dynamic range chemical UV dosimeter based on solvent cast polyvinyl chloride (PVC), J. Photochem. Photobiol. B Biol. 128 (2013) 92-99. doi:10.1016/j.jphotobiol.2013.09.001.

[12] A. Amar, A. V. Parisi, Spectral response of solvent-cast polyvinyl chloride (PVC) thin film used as a long-term UV dosimeter, J. Photochem. Photobiol. B Biol. 125 (2013) 115-120. 
doi:10.1016/j.jphotobiol.2013.05.012.

[13] P.W. Schouten, A. V. Parisi, D.J. Turnbull, Field calibrations of a long-term UV dosimeter for aquatic UVB exposures, J. Photochem. Photobiol. B Biol. 91 (2008) 108-116. doi:10.1016/j.jphotobiol.2008.02.004.

[14] M. Kozicki, E. Sasiadek, Textile UV detector with 2,3,5-triphenyltetrazolium chloride as an active compound, Radiat. Meas. 46 (2011) 510-526. doi:10.1016/j.radmeas.2011.03.016.

[15] K. Parambath Kootery, H. Jiang, S. Kolusheva, T.P. Vinod, M. Ritenberg, L. Zeiri, R. Volinsky, D. Malferrari, P. Galletti, E. Tagliavini, R. Jelinek, Poly(methyl methacrylate)supported polydiacetylene films: Unique chromatic transitions and molecular sensing, ACS Appl. Mater. Interfaces. 6 (2014) 8613-8620. doi:10.1021/am501414z.

[16] J. Hao, B. Xiong, X. Cheng, Y. He, E.S. Yeung, High-throughput sulfide sensing with colorimetric analysis of single Au-Ag core-shell nanoparticles, Anal. Chem. 86 (2014) 4663-4667. doi:10.1021/ac500376e.

[17] C. Fenzl, S. Wilhelm, T. Hirsch, O.S. Wolfbeis, Optical Sensing of the lonic Strength Using Photonic Crystals in a Hydrogel Matrix, ACS Appl. Mater. Interfaces. 5 (2013) 173178. doi:10.1021/am302355g.

[18] A. Kovács, M. Baranyai, L. Wojnárovits, I. Slezsák, W.L. McLaughlin, A. Miller, A. Moussa, Dose determination with nitro blue tetrazolium containing radiochromic dye films by measuring absorbed and reflected light, Radiat. Phys. Chem. 57 (2000) 711-716. doi:10.1016/S0969-806X(99)00501-0.

[19] H. Zheng, L. Jiang, H. Lou, Y. Hu, X. Kong, H. Lu, Application of Artificial Neural Network (ANN) and Partial Least-Squares Regression (PLSR) to predict the changes of anthocyanins, ascorbic acid, total phenols, flavonoids, and antioxidant activity during storage of red bayberry juice based on fractal ana, J. Agric. Food Chem. 59 (2011) 592600. doi:10.1021/jf1032476.

[20] M.E. Lee, A.M. Armani, Flexible UV Exposure Sensor Based on UV Responsive Polymer, 
ACS Sensors. 1 (2016) 1251-1255. doi:10.1021/acssensors.6b00491.

[21] P.S. Khiabani, A.H. Soeriyadi, P.J. Reece, J.J. Gooding, Paper-Based Sensor for Monitoring Sun Exposure, ACS Sensors. 1 (2016) 775-780.

doi:10.1021/acssensors.6b00244.

[22] D. Rosu, L. Rosu, C.N. Cascaval, IR-change and yellowing of polyurethane as a result of UV irradiation, Polym. Degrad. Stab. 94 (2009) 591-596.

doi:10.1016/j.polymdegradstab.2009.01.013.

[23] Learning Center for Research Essentials offered by Sigma-Aldrich. | Sigma-Aldrich, (n.d.). https://www.sigmaaldrich.com/chemistry/stockroom-reagents/learning-center.html (accessed August 5, 2018).

[24] weather forecast Europe - WeatherOnline, (n.d.). https://www.weatheronline.co.uk/weather/maps/city (accessed August 6, 2018).

[25] ASTM International - Standards Worldwide, (n.d.). https://www.astm.org/ (accessed August 6, 2018). 